\title{
Program Group on International Schools for Young Astronomers
}

\section{CHAIRPERSON: Michèle Gerbaldi \\ VICE-CHAIRPERSON: Ed Guinan}

Institut d'Astrophysique de Paris, Faculté des Sciences d'Orsay, France Villanova university, USA

\begin{abstract}
In the last triennium two International Schools for Young Astronomers took place in 2004 and 2005, respectively in Morocco and Mexico.
\end{abstract}

Keywords. Education

\section{Introduction}

Two International Schools for Young Astronomers (ISYA) took place during the period 2003-2006.

The 27th International School for Young Astronomers was organized at Al Akhawayn University in Ifrane (AUI), Morocco, under the auspice of Mr. Rachid Benmokhtar Benabdellah, President of Al Akhawayn University (July 02-23, 2004).

The Instituto Nacional de Astrofísica, Óptica y Electrónica (INAOE) in Tonantzintla, Puebla, Mexico, hosted the 28th International School for Young Astronomers from the 25 th of July to the 12th of August 2005. The school was financially supported primarily by the IAU and INAOE, with an additional contribution from the Instituto de Astronomía de la Universidad Nacional Autónoma de México (IA-UNAM).

\section{27th ISYA, 2004, Morocco}

This ISYA was organized after that a fist "Teaching for Astronomy Development" (TAD) program has been running since 1999 at the University Hassan II in Casablanca, Morocco. During this ISYA a second TAD agreement has been signed by the International Astronomical Union (IAU) represented by its general secretary Prof. Oddbjorn Engvold and AUI President Mr. Rachid Benmokhtar Benabdellah. Prof. James C. White (TAD chairperson) also attended the ceremony. This new agreement has the objective to support the continued, long-term development of astronomy and astrophysics in Morocco.

\subsection{ISYA Committee}

The members of the Programme Committee were:

Michèle Gerbaldi (Institut d'Astrophysique, Paris, France)

Hassane Darhmaoui (Al Akhawayn University)

Khalil Chamcham (Casablanca University, Oxford University, UK)

Amine Bensaid (Al Akhawayn University, School of Science \& Engineering) was the Chairperson of the Local Organizing Committee. 


\subsection{ISYA Participants}

The number of participants was 29 (female: 9, male: 20) 11 Moroccan and 18 foreigners from: Algeria, Bulgaria, Iran, Jordan, Lebanon, Macedonia, Malaysia, Nigeria, Palestine, South Africa, Sudan and Turkey. The background of the participants, ranged from finishing their BSci. degree to having started their PhD about one year ago.

\subsection{ISYA Academic Programme}

The faculty members and lectures were:

- Bruce Partridge (Haverford College, US), Radio Astronomy and Cosmology.

- Bruno Guiderdoni (Institut d'Astrophysique de Paris, France), Galaxy Formation.

- Edward Guinan (Villanova University, USA), Magnetic Activity of the Sun and Solar type Stars. Variable and Eclipsing Stars as Astrophysical Laboratories.

- Ignasi Ribas (Barcelona university, Spain), Astronomical techniques, data analysis.

- Jean-Pierre de Greve (Brussels University, Belgium), Evolution of close binary stars.

- Joseph Silk (Oxford University, UK), Galaxy Formation.

- Kavilan Moodley (University of KwaZulu-Natal, South Africa), Cosmological Models.

- Mariano Mendez (SRON - National Institute for Space Research, The Netherland), High-Energy Astrophysics.

- Martin Hendry (University of Glasgow, UK), Statistical Astronomy.

- Michèle Gerbaldi (Institut d'Astrophysique de Paris and Université de Paris-Sud Orsay, France), Stellar Atmosphere.

- Mohammed Badaoui (APESA- Agronomic and Veterinary Institute Hassan II, Rabat - Morocco), Infrared high resolution molecular spectroscopy.

- Pedro G. Ferreira(Oxford University, UK), Cosmology with the Cosmic Microwave Background.

Sessions were organized for talks by the participants on their current interest and research. More than 15 talks were given.

Thanks to a fruitful collaboration with the AUI Information Technology Services department a network of 20 computers under LINUX was set up for the practical activities. Specialized software packages were installed for the reduction and analysis of data, (IRAF) and (XSPEC) among others. More than 25 hours were spent in data reduction and analysis

The Al Jabr School, in Casablanca, is acknowledged for the loan to AUI, of the $20 \mathrm{~cm}-$ telescope, allowing observational sessions to be set up.

This ISYA could be organized thanks to the financial support of the IAU, the Al Akhawayn university; ICTP (The Abdu Salam International Centre for Theoretical Physics, Italy) and CNRST (Centre National pour la Recherche Scientifique et Technique, Morocco) are acknowledged for the grants given for the venue of this event.

ISYA 27th also opened its doors to interested AUI students and faculty members who attended some of its activities.

\section{28th ISYA, 2005, Mexico}

\subsection{ISYA Committee}

The 28th ISYA was a great success due to the organization and careful planning by the Local Organizing Committee (LOC) and the general support offered by many departments at INAOE including the computing technical department, the Public Relations Office, and the secretary of the Guillermo Haro Programme at INAOE. 
The LOC consisted of :

Itziar Aretxaga (INAOE - LOC chair)

Alberto Carramiñana (INAOE - Head of the Astrophysics Dept.)

Esperanza Carrasco (INAOE)

José Franco (IA-UNAM - Director)

José Guichard (INAOE - General Director)

Francisco Soto (INAOE - Research and Teaching Director)

\subsection{ISYA Participants}

A total of 154 applications to attend the school were received, out of which 47 were selected from the following countries: 20 (6 female, 14 male) from Brazil, Chile, Colombia, Cuba, Honduras, Peru, Salvador, Trinidad and Tobago, and Venezuela; and 27 (11 female, 16 male) from Mexican institutions (IA-UNAM Ensenada, Universidad de Guanajuato, CRyA-UNAM, IA-UNAM Mexico City, INAOE and Universidad de Sonora). The students had very mixed academic backgrounds and experience ranging from final-year undergraduates to $\mathrm{PhD}$ students.

Although English is the official language of the ISYA, the majority of students were Spanish-speaking. The Latin American ISYA students however observed the policy to speak English to ensure that their fellow students from Brazil and Trinidad \& Tobago were not excluded from both casual conversations and more formal discussions.

\subsection{ISYA Academic Programme}

The faculty members and lectures were:

- Rodolfo Barbá, Universidad de La Serena, Chile, Astronomical Databases and Virtual Observatories: Surfing the Tsunami of Data.

- Enrique Gaztañaga, Institut d'Estudis Espacials de Catalunya, Spain, Cosmology and Large-Scale Structure.

- Michèle Gerbaldi, Institut d'Astrophysique de Paris, France, Stellar Atmospheres: from Observations to Theoretical Challenges.

- Edward Guinan, University of Villanova, USA, Extra-solar Planets: Properties and Future Possibilities.

- Rafael Guzmán. University of Florida, USA, The Universe According to OpticallySelected Galaxies.

- David Hughes, INAOE, Mexico, The Hidden Universe Revealed at Millimetre Wavelengths.

- Susana Lizano, Universidad Nacional Autónoma de México, Mexico, Theory of Galactic Star Formation.

- Claudia Mendes de Oliveira, Universidade de São Paulo, Brazil, Groups and Clusters of Galaxies.

- Manuel Peimbert, Universidad Nacional Autónoma de México, Mexico, The Chemical History of the Universe.

- Massimo Turatto, Osservatorio di Padova, Italy, Supernovae: from Stellar Evolution to Cosmology.

- Guillermo Tenorio-Tagle, INAOE, Mexico,

Feedback and Hydrodynamics of the Interstellar Medium.

- Elena Terlevich and Vahram Chavushyan, INAOE, Mexico, Optical/IR Observational Techniques.

- William Wall and David Hughes, INAOE, Mexico, Millimetre-Wavelength Observational Techniques. 
Three of these topics were designed to be taught through practical laboratories, with more than 30 hours devoted to data mining, reduction and analysis. These practical classes were based on access to real data and fully-equipped PCs with all the necessary data-reduction and image processing software pre-installed. The students had 24-hour daily access to the PCs. As part of the practical assignments, the students also had to conduct optical observations with the $1 \mathrm{~m}$ telescope of the Observatorio Astronómico Nacional in Tonantzintla (which is operated by IA-UNAM on an adjacent campus to INAOE).

In addition to the practical classes, several scientific tours were also arranged during the ISYA. During the 3 -week school groups of about 20 students and lecturers rotated visits to the Large Millimetre Telescope (LMT), which is operated by INAOE and the University of Massachussets in Amherst (USA) and which is currently being built on top of Sierra Negra at an elevation of $4600 \mathrm{~m}$, and also to the INAOE Schmidt Camera and Solar Telescope, and to the INAOE optical aspheric laboratory and workshop.

The ISYA students were encouraged to describe their current research interests in poster format: 35 scientific posters were presented, from which 8 were selected to give 15-min short oral presentations during a general session.

\section{Acknowledgements}

We would like to acknowledge Oddbjorn Engvold, General Secretary, and the IAU Executive Committee members for their strong support to the ISYA Programme 